\title{
SSMFN: A fused spatial and sequential deep learning model for methylation site prediction
}

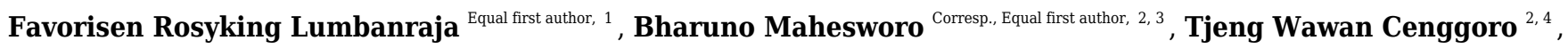 \\ Digdo Sudigyo ${ }^{2}$, Bens Pardamean ${ }^{2,5}$ \\ ${ }^{1}$ Department of Computer Science, Faculty of Mathematics and Natural Science, University of Lampung, Bandar Lampung, Lampung, Indonesia \\ 2 Bioinformatics and Data Science Research Center, Bina Nusantara University, West Jakarta, Jakarta, Indonesia \\ 3 Statistics Departement, School of Computer Science, Bina Nusantara University, West Jakarta, Jakarta, Indonesia \\ 4 Computer Science Departement, School of Computer Science, Bina Nusantara University, West Jakarta, Jakarta, Indonesia \\ 5 Computer Science Department, BINUS Graduate Program - Master of Computer Science, Bina Nusantara University, West Jakarta, Jakarta, Indonesia \\ Corresponding Author: Bharuno Mahesworo \\ Email address: bharuno.mahesworo@binus.edu
}

Background: Conventional in vivo method for post-translational modification site prediction such as spectrophotometry, Western Blotting, and chromatin immune precipitation can be very expensive and time-consuming. Neural networks (NN) is one of the computational approaches that can predict effectively the post-translational modification site. We developed a neural network model namely Sequential and Spatial Methylation Fusion Network (SSMFN) to predict possible one of post-translational modification process locations, methylation, site on the protein sequence.

Method: We designed our model to be able to extract spatial and sequential information from amino acid sequences. Convolutional Neural Networks (CNN) is applied to harness spatial information, while Long Short-Term Memory (LSTM) is applied for sequential data. The latent representation of the CNN and LSTM branch are then fused. Afterwards, we compared the performance of our proposed model to the state-ofthe-art methylation site prediction models on the balanced and imbalanced dataset.

Results: Our model appeared to be better in almost all measurement when trained on the balanced training dataset. On the imbalanced training dataset, all of the models gave better performance since they are trained on more data. In several metrics, our model also surpasses the PRMePred model, which requires a laborious effort for feature extraction and selection.

Conclusion: Our models achieved the best performance across different environments in almost all measurements. Also, our result suggests that the NN model trained on a balanced training dataset and tested on an imbalanced dataset will offer high specificity and low sensitivity. Thus, the NN model for methylation site prediction should be trained on an imbalanced dataset. Since in the actual application, there are far more negative samples than positive samples. 


\title{
SSMFN: A Fused Spatial and Sequential Deep Learning Model for Methylation Site Prediction
}

\author{
Favorisen Rosyking Lumbanraja ${ }^{1, *}$, Bharuno Mahesworo, 2,3, , Tjeng \\ Wawan Cenggoro $^{2,4}$, Digdo Sudigyo ${ }^{2}$, and Bens Pardamean ${ }^{2,5}$
${ }^{1}$ Department of Computer Science, Faculty of Mathematics and Natural Science, University of Lampung, Bandar Lampung, Indonesia
${ }^{2}$ Bioinformatics Data Science Research Center, Bina Nusantara University, West Jakarta, Indonesia
${ }^{3}$ Statistics Department, School of Computer Science, Bina Nusantara University, Jakarta, Indonesia
${ }^{4}$ Computer Science Department, School of Computer Science, Bina Nusantara
University, Jakarta, Indonesia
${ }^{5}$ Computer Science Department, BINUS Graduate Program - Master of Computer
Science, Bina Nusantara University, Jakarta, Indonesia
*Equal Authorship \\ Corresponding author: \\ Bharuno Mahesworo 2,3 \\ Email address: bharuno.mahesworo@binus.edu
}

\section{ABSTRACT}

\begin{abstract}
Background: Conventional in vivo methods for post-translational modification site prediction such as Mass Spectrophotometry, Western Blotting, and Chromatin Immune Precipitation can be very expensive and time-consuming. One of the solutions for a faster prediction is by using computational approaches, for instance, with machine learning algorithms such as Neural Network (NN). In this study, we developed a neural network model named Sequential and Spatial Methylation Fusion Network (SSMFN) to predict possible one of post-translational modification process locations, methylation, site on the protein sequence.

Method: We designed our model to be able to extract spatial and sequential information from amino acid sequences. Convolutional Neural Network (CNN) is applied to harness spatial information, while Long Short-Term Memory (LSTM) is applied for sequential data. Our model predicts methylation site from the fused latent representation of the CNN and LSTM branches. To evaluate the performance of our proposed model, we compared it to the state-of-the-art methylation site prediction models on the balanced and imbalanced dataset.

Results: Our model outperformed the previous state-of-the-art NN models in almost all metrics when trained on the balanced training dataset. Moreover, our model is also the best NN model on the imbalanced training dataset, which is the dataset with the actual distribution in nature. It also has competitive performance compared to the PRMePred model, which requires a laborious effort for feature extraction and selection.

Conclusion: The proposed model achieved the best performance across different environments in almost all metrics. Additionally, we observed that the models that were trained on the balanced dataset have low performance (high specificity and low sensitivity) on the test dataset that was imbalanced. On the other hand, trained on the imbalanced dataset, the models achieved higher performance on the test dataset. This result suggested that we need to train methylation site prediction models with an imbalanced dataset. Because, in nature, the distribution of the samples is imbalanced (there are far more negative samples than positive samples).
\end{abstract}




\section{INTRODUCTION}

Methylation is a post-translational modification (PTM) process that modifies the functional and conformational changes of a protein. The addition of a methyl group to the protein structure plays a role in the epigenetic process, especially in histones (Lee et al., 2005). Histone methylation in Arginine (R) and Lysine (K) residues substantially affects the level of gene expression along with other PTM processes such as acetylation and phosphorylation (Schubert et al., 2006). Moreover, methylation directly alters the regulation, transcription, and structure of chromatin (Bedford and Richard, 2005). Genetic alterations through the methylation process induce oncogenes and tumor suppressor genes that play a crucial role in carcinogenesis and metastasis cancer (Zhang et al., 2019).

Currently, most of the methods for PTM sites prediction were conducted by implementing in vivo methods, such as Mass Spectrophotometry, Western Blotting, and Chromatin Immune Precipitation (ChIP). However, computational (in silico) approaches are starting to be more popular for PTM sites prediction, especially methylation. Computational approaches for predicting protein methylation sites can be an inexpensive, highly accurate, and fast alternative method through massive data sets. The commonly used computational approaches are Support Vector Machine (SVM) (Chen et al., 2006; Shao et al., 2009; Shien et al., 2009; Shi et al., 2012; Lee et al., 2014; Qiu et al., 2014; Wen et al., 2016), Group-Based Prediction System (GPS) (Deng et al., 2017), random forest (Wei et al., 2017), and Neural Network (NN) (Chen et al., 2018; Hasan and Khatun, 2018; Chaudhari et al., 2020).

The application of the machine learning approach to predict possible methylation sites on protein sequences has been studied in numerous previous research. The latest and the most relevant studies to our study were conducted by Chen et al. (2018) and Chaudhari et al. (2020). Chen et al. (2018) developed MUscADEL (Multiple Scalable Accurate Deep Learner for lysine PTMs), a methylation site prediction model that was trained and tested on human and mice protein data sets. MUscADEL utilized bidirectional Long Short Term Memory (LSTM) (Graves and Schmidhuber, 2005). Meanwhile, Chen et al. (2018) hypothesized that the order of amino acids in the protein sequence has a significant influence on the location where the methylation process can occur. The other model is DeepRMethylSite which was developed by Chaudhari et al. (2020). The model was implemented with the combination of Convolutional Neural Network (CNN) and LSTM. The combination was expected to be able to extract the spatial and sequential information of the amino acids sequences.

Before the practical application by Chaudhari et al. to predict methylation site, a combination of LSTM and CNN approach has been implemented since 2015 by Xu et al. (2015) to strengthen a face recognition model. This combination was also found In the natural language processing (NLP) area. For instance, Jin Wang (2016) developed a Dimensional Sentiment Analysis model and suggested that a combination of LSTM and CNN is capable of capturing long-distance dependency and local information patterns. Related to NLP, Chuhan Wu (2018) developed an LSTM-CNN model with similar architecture to other previous studies where the CNN layer and LSTM layer were implemented in serial structure. Recently, the combination of CNN and LSTM was also applied for educational data (Prabowo et al., 2021).

In this study, we developed the Sequential and Spatial Methylation Fusion Network (SSMFN) to predict possible methylation sites on the protein sequence. Similar to DeepRMethylSite, SSMFN also utilized CNN and LSTM. However, instead of treating them as an ensemble model, we fused the latent representation of the CNN and LSTM modules. By allowing more relaxed interaction between the CNN and LSTM modules, we hypothesized that the fusion approach can extract better features than the model with the ensemble approach.

\section{METHODS}

\section{Dataset}

The dataset in this study was obtained from the previous methylation site prediction study by Kumar et al. (2017). The data was collected from other studies as well as from Uniprot protein database (Apweiler et al., 2004). The collected data was furthermore experimentally verified in vivo.

The dataset comprises sequences of 19 amino acids with Arginine in the middle of the sequence because the possible location for methylation is on Arginine (R). These sequences are segments from the full amino acids sequence. Examples of the amino acids sequences in this dataset are shown in Table 1. The dataset was split into three datasets: training, validation, and independent dataset. Each dataset 
contains positive and negative samples, where positive samples are the sequence where methylation occurs in the middle amino acid. Because the original dataset was imbalanced, previous studies often constructed a new balanced dataset to improve the performance of their model. This practice is needed because most machine learning methods are not robust to imbalanced training data. Following the typical practice in previous studies, we also created a balanced training dataset as well as a balanced validation dataset for a fair comparison.

\begin{tabular}{|c|c|c|c|c|c|c|c|c|c|c|c|c|c|c|c|}
\hline \multirow[t]{2}{*}{ No } & \multicolumn{15}{|c|}{ Sequence } \\
\hline & $1 \mathrm{st}$ & 2nd & $3 \mathrm{rd}$ & & . & 8th & 9th & 10th & 11th & 12 th & . & . & 17th & 18th & 19th \\
\hline 1 & V & $\mathrm{E}$ & $\mathrm{S}$ & . & . & V & $\mathrm{T}$ & $\mathbf{R}$ & $\mathrm{L}$ & $\mathrm{H}$ & . & . & $\mathrm{H}$ & $\mathrm{M}$ & $\mathrm{N}$ \\
\hline 2 & $\mathrm{~K}$ & $\mathrm{~N}$ & $\mathrm{H}$ & . & . & I & $\mathrm{S}$ & $\mathbf{R}$ & $\mathrm{H}$ & $\mathrm{H}$ & . & . & $\mathrm{D}$ & $\mathrm{P}$ & Q \\
\hline 3 & $\mathrm{H}$ & $\mathrm{P}$ & $\mathrm{P}$ & . & . & $\mathrm{R}$ & $\mathrm{L}$ & $\mathbf{R}$ & $\mathrm{G}$ & I & . & . & W & $\mathrm{D}$ & $\mathrm{H}$ \\
\hline$\cdot$ & - & . & . & . & . & . & . & - & . & . & . & . & . & . & . \\
\hline . & . & . & . & . & . & . & . & . & . & . & . & . & . & . & . \\
\hline $\mathrm{n}$ & $\bar{R}$ & $\mathrm{~S}$ & I & . & . & $\bar{A}$ & $\mathrm{C}$ & $\overline{\mathbf{R}}$ & I & $\mathrm{R}$ & . & . & $\mathrm{K}$ & $\mathrm{W}$ & $\mathrm{Y}$ \\
\hline
\end{tabular}

Table 1. Protein Sequence Dataset Example

\begin{tabular}{l|l|r}
\hline Data class & Label & n sequences \\
\hline \multirow{2}{*}{ Training } & Positive & 1038 \\
\cline { 2 - 3 } & Negative & 5190 \\
\hline \multirow{2}{*}{ Balanced Training } & Positive & 1038 \\
\cline { 2 - 3 } & Negative & 1038 \\
\hline \multirow{2}{*}{ Balidation } & Positive & 1131 \\
\cline { 2 - 3 } & Negative & 3033 \\
\hline \multirow{2}{*}{ Independent $($ Test $)$} & Positive & 1131 \\
\cline { 2 - 3 } & Negative & 1131 \\
\cline { 2 - 3 } & Positive & 260 \\
\cline { 2 - 3 } & Negative & 260 \\
\hline
\end{tabular}

Table 2. Amino Acids Sequences Dataset List.

\section{Experiment}

Firstly, to understand the contribution of each element in the proposed model, we carried an ablation study on our proposed model. The elements tested and explored in this ablation study were the CNN and LSTM branches of the model. Afterward, we compared the performance of our proposed model to DeepRMethylSite (Chaudhari et al., 2020). Additionally, we also provided a comparison to a standard multi-layer perceptron model. To measure the effect of the data distribution (balanced or imbalanced), we conducted separate experiments for the balanced and the original imbalanced dataset. Afterward, the trained models from both experiments were validated and tested on the balanced validation dataset, the imbalanced validation dataset, and the test dataset, respectively. The workflow of this study is illustrated in Figure 1. All models in the experiment were developed using Python machine learning library, PyTorch (Paszke et al., 2019). To train the models, we utilized a NVIDIA Tesla P100 Graphical Processing Unit (GPU) as well as a publicly available GPU instance provided by Google Colab.

\section{Spatial and Sequential Methylation Fusion Network (SSMFN)}

Our proposed model, Spatial and Sequential Methylation Fusion Network (SSMFN), was designed with the motivation that a protein sequence can be perceived as both spatial and sequential data. The view of a protein sequence as spatial data assumes that the amino acids are arranged in a one-dimensional space. On the other hand, protein sequence can also be thought of as sequential data by assuming that the next amino acid is the next time step of particular amino acid. On modelling protein sequences with deep learning, CNN is applied when adopting spatial data view, while LSTM is applied for the sequential data. Using the information from both views has been shown to be beneficial by Chaudhari et al. (2020). Their model was implemented by having an ensemble model of CNN and LSTM that read the same sequence. However, 


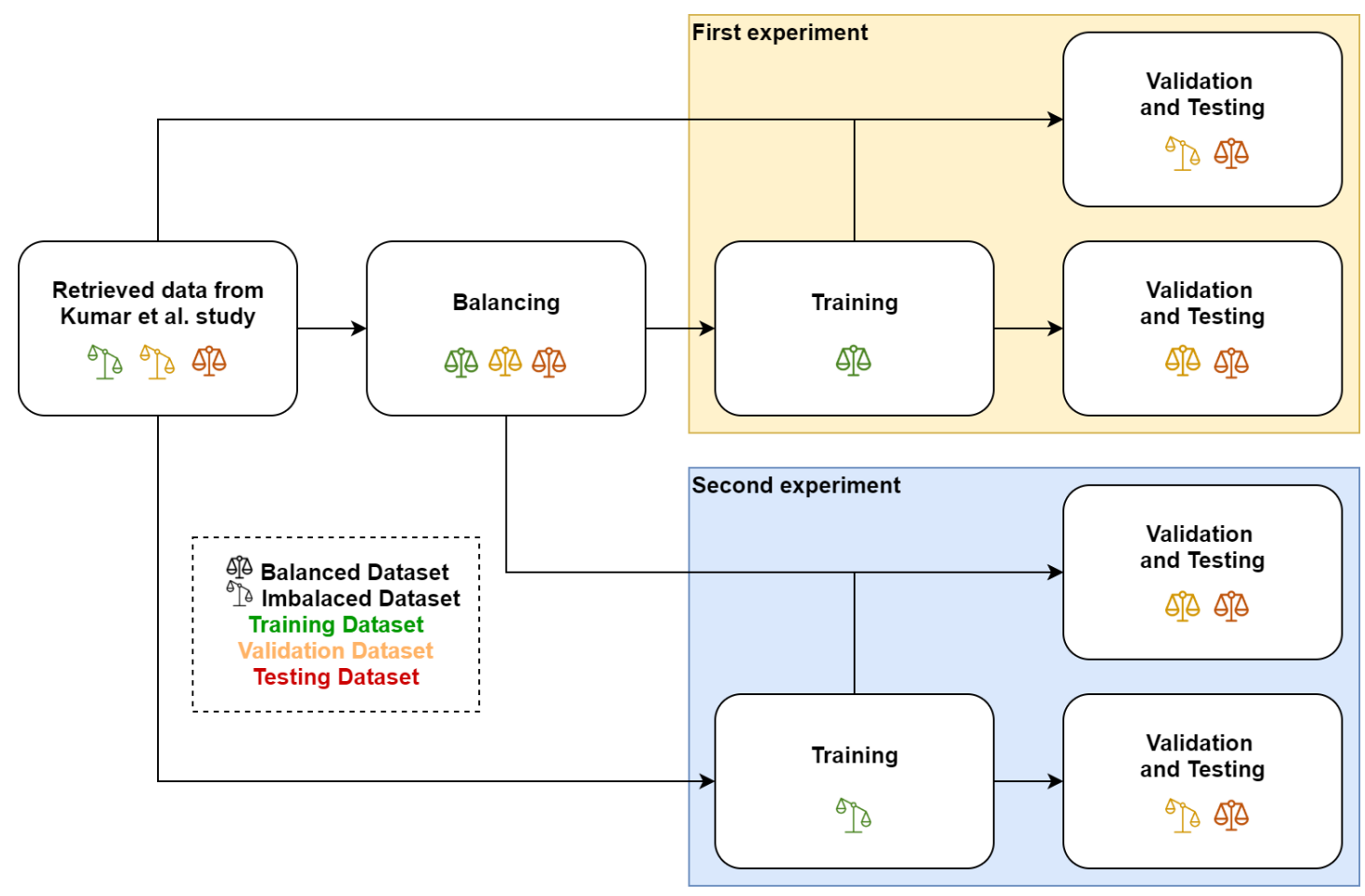

Figure 1. Research workflow. The chart shows that the data we used in this research was retrieved from Kumar et al. (2017) study. The data was afterward balanced accordingly. In the first experiment, we trained our model using the balanced training dataset. Subsequently, we validated and tested the model on the balanced and the imbalanced dataset. We did a similar workflow for the second experiment. However, instead of the balanced dataset, we trained the model on the imbalanced training dataset.

Chaudhari et al. processed the spatial and sequential view with separate sub-models. As a consequence, it cannot extract joint spatial-sequential features, which might be beneficial in modelling protein sequences. Having observed that, we constructed SSMFN as a deep learning model with an architecture that can fuse the latent representation of CNN modules and LSTM modules.

To read the amino acid sequence, SSMFN applied an embedding layer with 21 neurons. This embedding layer was used to enhance the expression of each amino acid. Thus, the number of neurons in this layer matches the amounts of amino acids variants. Therefore, each type of amino acid can have a different vector representation. The output of this layer is then split into LSTM and CNN branches. In the LSTM branch, we created two LSTM layers with 64 neurons each. Every LSTM layer is followed by a dropout layer with a 0.5 drop rate. It is subsequently followed by a fully connected layer at the end of the branch with 32 neurons. This fully connected layer serves as a latent representation generator that is fused with the latent representation from the CNN branch.

In contrast, the CNN branch comprised four CNN layers with 64 neurons in each layer. Unlike the LSTM layers, residual connections were utilized in the CNN branch. Each CNN layer is a 2D convolutional layer with rectified linear units (ReLU) as the activation function. Every CNN layer also has a $2 \mathrm{D}$ batch normalization layer and a dropout layer which is set at 0.5 . At the end of the branch, a fully connected layer with 32 neurons is installed to match the output with the LSTM branch.

In the next step, the latent representation of both branches was fused with a summation operation. The fused representation was subsequently processed through a fully connected layer with 2 neurons as the last layer. This layer predicts whether the methylation occurred at the center of the amino acid or not. The architecture of the proposed model and the hyperparameter settings is illustrated in Figure 2 and listed in Table 3. The code of this model can be found in this following link https://github.com/bharuno/SSMFNMethylation-Analysis. 


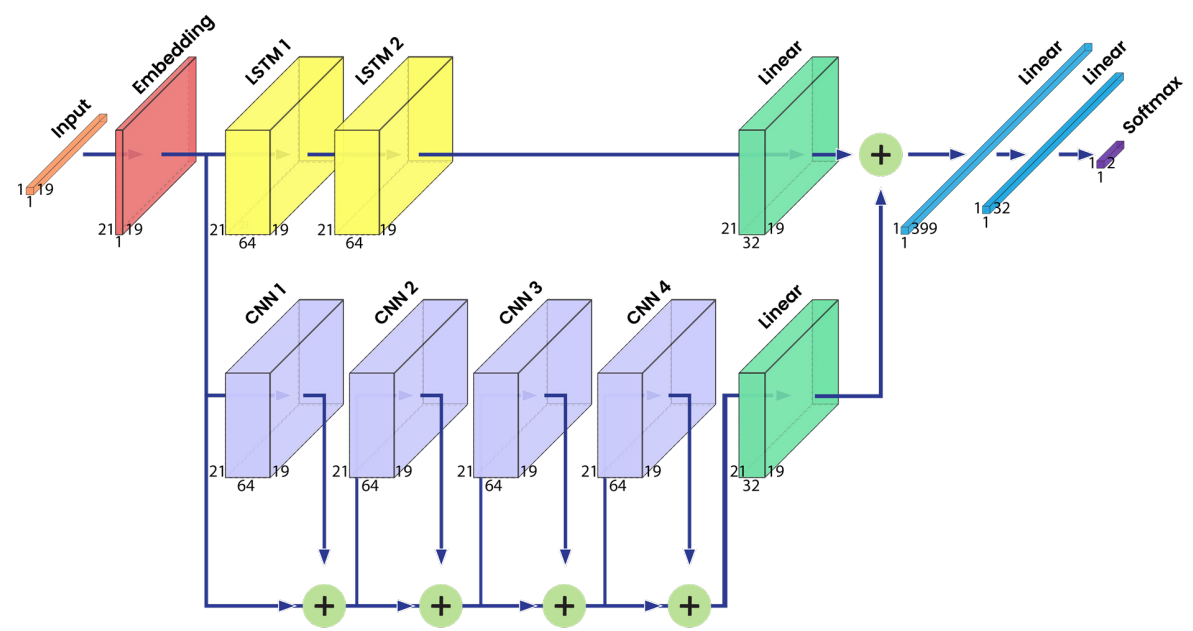

Figure 2. Proposed Neural Network Architecture.

\section{Comparison to a Standard Multi-Layer Perceptron}

A standard multi-layer perceptron (SMLP) NN was developed to be compared to our proposed model. This multi-layer perceptron model was included in this study to provide an insight into the performance of a simple model to solve the methylation site prediction problem. This model consists of an embedding layer followed by two fully connected layers. The embedding layer has 21 neurons because there are 21 types of amino acids. The first fully connected layer has 399 neurons which came from 21 (types of amino acid) multiplied by 19 (protein sequence length). After the first layer, we put a second fully connected layer that has two neurons as the output for prediction. The structure of this model is shown in Figure 3.

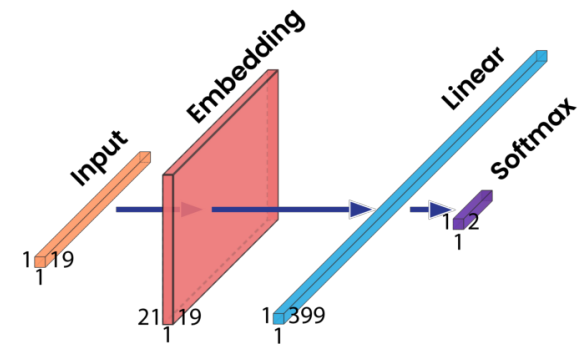

Figure 3. The Standard Multi-Layer Perceptron Architecture.

\section{Comparison to DeepRMethylSite}

For a fair comparison of our proposed model to other state-of-the-art methylation site prediction models, we re-conducted the experiment to train DeepRMethylSite (Chaudhari et al., 2020) with the same dataset used by our proposed model. To obtain optimal DeepRMethylSite performance on our dataset, we adjusted several hyperparameters. Firstly, we changed the LSTM branch optimizer, from Adadelta to Adam. Secondly, we removed recurrent dropout layers in the LSTM branch. Finally, we set the maximum number of epochs to 500 .

\section{Evaluation}

To evaluate the performance of the proposed model and to compare it to the models from previous studies, we utilized Accuracy (Equation 1), Sensitivity (Equation 2), Specificity (Equation 3), F1 score (Equation 


\begin{tabular}{l|l}
\hline Parameter & Settings \\
\hline Learning rate & 0.001 \\
\hline Epochs & 500 \\
\hline Optimizer & Adam \\
\hline Embedding layer neurons & 21 \\
\hline Embedding layer output dimension & $21 \times 19=399$ \\
\hline Output layer neurons & 2 \\
\hline \multicolumn{2}{c}{ LSTM Branch } \\
\hline LSTM layers neurons & 64 \\
\hline Dropout layers drop rate & 0.5 \\
\hline Fully connected layer neurons & 32 \\
\hline \multicolumn{2}{c}{ CNN Branch } \\
\hline CNN layers neurons & 64 \\
\hline CNN layers activation function & Rectified Linear Units \\
\hline Dropout layers drop rate & 0.5 \\
\hline Fully connected layer neurons & 32 \\
\hline
\end{tabular}

Table 3. Hyperparameter Settings.

4), Matthews Correlation Coefficient (MCC) (Equation 5), and Area Under Curve (AUC) (Bradley, 1997). These metrics were commonly employed in the previous research with a focus on prediction protein phosphorylation site (Lumbanraja et al., 2018, 2019). The AUC was computed using the scikit-learn library from the Receiver Operating Characteristic (ROC) of the models' performance.

$$
\text { Accuracy }=\frac{T P+T N}{T P+T N+F P+F N}
$$

$$
\text { Sensitivity }=\frac{T P}{T P+F N}
$$

$$
\text { Specificity }=\frac{T N}{T N+F P}
$$

$$
F 1 \text { score }=\frac{T P}{T P+F P+F N}
$$

$$
M C C=\frac{(T P * T N)-(F P * F N)}{\sqrt{(T P+F P)(T P+F N)(T N+F P)(T N+F N)}}
$$

\section{RESULTS}

Table 4 and Table 5 show the results obtained from our ablation study. Meanwhile, Table 6 and Table 7 summarized the comparative results of our model to the previous models with the balanced and imbalanced training dataset, respectively. In Table 6, we also added the performance of several methylation site prediction models from previous studies including MeMo (Chen et al., 2006), MASA (Shien et al., 2009), BPB-PPMS (Shao et al., 2009), PMeS (Shi et al., 2012), iMethylPseAAC (Qiu et al., 2014), PSSMe (Wen et al., 2016), MePred-RF (Wei et al., 2017) and PRmePRed (Kumar et al., 2017). The performances of MeMo, MASA, BPB-PPMS, PMeS, iMethylPseAAC, PSSMe and MePred-RF were reported by Chaudhari et al. (2020). Meanwhile, the performance of PRmePRed was reported by Kumar et al. (2017).

\section{DISCUSSION}

The results of the ablation study in Table 4 and Table 5 show that the LSTM branch and CNN branch achieved better performance compared to the merged model at least on one dataset. However, the merged 


\begin{tabular}{l|l|l|l|l|l|l}
\hline Model & Acc & F1 & Sens & Spec & MCC & AUC \\
\hline \multicolumn{7}{c}{ Validated on The Imbalanced Validation Dataset } \\
\hline SSMFN CNN & 0.7891 & 0.7649 & 0.5745 & 0.9368 & 0.5649 & 0.8120 \\
\hline SSMFN LSTM & $\mathbf{0 . 8 2 5 2}$ & $\mathbf{0 . 7 9 8 5}$ & $\mathbf{0 . 6 3 2 8}$ & 0.9354 & $\mathbf{0 . 6 1 4 8}$ & 0.8326 \\
\hline SSMFN Merged & 0.8187 & 0.7943 & 0.6175 & $\mathbf{0 . 9 4 4 2}$ & 0.6143 & $\mathbf{0 . 8 3 5 9}$ \\
\hline \multicolumn{7}{c}{ Validated on The Balanced Validation Dataset } \\
\hline SSMFN CNN & $\mathbf{0 . 8 4 3 1}$ & $\mathbf{0 . 8 4 2 7}$ & $\mathbf{0 . 8 7 6 7}$ & 0.8149 & $\mathbf{0 . 6 8 8 9}$ & 0.8120 \\
\hline SSMFN LSTM & 0.8302 & 0.3020 & 0.8195 & 0.8417 & 0.6609 & 0.8326 \\
\hline SSMFN Merged & 0.8360 & 0.8358 & 0.8130 & $\mathbf{0 . 8 6 2 6}$ & 0.6738 & $\mathbf{0 . 8 3 5 9}$ \\
\hline \multicolumn{7}{c}{ Tested on The Test Dataset } \\
\hline SSMFN CNN & 0.7962 & 0.7960 & $\mathbf{0 . 8 1 0 5}$ & 0.7831 & 0.5929 & 0.7962 \\
\hline SSMFN LSTM & 0.7981 & 0.7980 & 0.8063 & 0.7903 & 0.5964 & 0.7981 \\
\hline SSMFN Merged & $\mathbf{0 . 8 1 1 5}$ & $\mathbf{0 . 8 1 1 5}$ & 0.8000 & $\mathbf{0 . 8 2 4 0}$ & $\mathbf{0 . 6 2 3 5}$ & $\mathbf{0 . 8 1 1 5}$ \\
\hline
\end{tabular}

Table 4. The First Ablation Study, Trained on the Balanced Training Dataset.

\begin{tabular}{l|l|l|l|l|l|l}
\hline Model & Acc & F1 & Sens & Spec & MCC & AUC \\
\hline \multicolumn{7}{c}{ Validated on The Imbalanced Validation Dataset } \\
\hline SSMFN CNN & 0.8939 & 0.8502 & $\mathbf{0 . 9 3 8 9}$ & 0.8834 & 0.7230 & 0.8179 \\
\hline SSMFN LSTM & $\mathbf{0 . 9 1 6 7}$ & $\mathbf{0 . 8 8 9 1}$ & 0.9100 & $\mathbf{0 . 9 1 8 6}$ & $\mathbf{0 . 7 8 3 6}$ & $\mathbf{0 . 8 7 0 4}$ \\
\hline SSMFN Merged & 0.9078 & 0.8774 & 0.8895 & 0.9133 & 0.7598 & 0.8596 \\
\hline \multicolumn{7}{c}{ Validated on The Balanced Validation Dataset } \\
\hline SSMFN CNN & 0.7529 & 0.7372 & $\mathbf{0 . 9 9 4 8}$ & 0.6698 & 0.5798 & 0.8179 \\
\hline SSMFN LSTM & 0.8638 & 0.8624 & 0.9567 & $\mathbf{0 . 8 0 2 4}$ & $\mathbf{0 . 7 5 6 0}$ & $\mathbf{0 . 8 7 0 4}$ \\
\hline SSMFN Merged & $\mathbf{0 . 8 6 5 6}$ & $\mathbf{0 . 8 6 4 0}$ & 0.9672 & 0.8003 & 0.7491 & 0.8596 \\
\hline \multicolumn{7}{c}{ Tested on The Test Dataset } \\
\hline SSMFN CNN & 0.7404 & 0.7228 & $\mathbf{0 . 9 8 4 5}$ & 0.6598 & 0.5566 & 0.7404 \\
\hline SSMFN LSTM & 0.8442 & 0.8418 & 0.9590 & $\mathbf{0 . 7 7 5 4}$ & 0.7110 & 0.8442 \\
\hline SSMFN Merged & $\mathbf{0 . 8 4 6 2}$ & $\mathbf{0 . 8 4 3 5}$ & 0.9688 & 0.7744 & $\mathbf{0 . 7 1 7 3}$ & $\mathbf{0 . 8 4 6 2}$ \\
\hline
\end{tabular}

Table 5. The Second Ablation Study, Trained on the Imbalanced Training Dataset.

models achieved better performance in most of the datasets, specifically in the test dataset. This fact indicates that the merged model has a better generalization capability than the model with only CNN or LSTM branches.

In the experiment on the balanced training dataset, our proposed model emerged as the best $\mathrm{NN}$ model with the best performance in all metrics except sensitivity among all other NN models. Interestingly, the DeepRMethylSite final result (merged) was not better in all metrics compared to its CNN branch and its LSTM branch. On the imbalanced validation dataset, our proposed model, SSMFN, has more than 4\% higher accuracy and 6\% higher MCC which is the best parameter for assessing model performance on imbalanced data, compared to the DeepRMethylSite model. On the balanced validation dataset and test dataset, SSMFN has 2-4\% higher accuracy compared to DeepRMethylSite.

In Table 6, we also present the performance of other methylation site prediction models from previous studies as reported by Chen et al. (2018) and Chaudhari et al. (2020). The models from previous studies provided an overview of the performance of non-neural-network models. The best non-neural-network model, PRmePRed, has more than 5\% higher accuracy than SSMFN. However, it should be noticed that non-neural-network models require heavy feature engineering, which is also found in PRmePRed. This introduced unnecessary manual labor that can be avoided by the utilization of modern NN models, which are also known as deep learning. Interestingly, the SMLP model provided slightly better performance than DeepRMethylSite on the test dataset. This does not implicate that the SMLP model has a better performance compared to the DeepRMethylSite it has relatively poor performance in the validation dataset, both balanced and imbalanced.

When trained on the balanced training dataset and tested on the imbalanced validation dataset, most of the models have high specificity and low sensitivity. This phenomenon is normal since the training and 


\begin{tabular}{|c|c|c|c|c|c|c|}
\hline Model & Acc & F1 & Sens & Spec & MCC & AUC \\
\hline \multicolumn{7}{|c|}{ Validated on The Imbalanced Validation Dataset } \\
\hline DeepRMethylSite CNN & 0.7819 & 0.7557 & 0.5668 & 0.9259 & 0.5428 & 0.7990 \\
\hline DeepRMethylSite LSTM & 0.7699 & 0.7479 & 0.5480 & 0.9394 & 0.5430 & 0.8024 \\
\hline DeepRMethylSite Merged & 0.7743 & 0.7518 & 0.5474 & 0.9394 & 0.5481 & 0.8021 \\
\hline SMLP & 0.7209 & 0.7018 & 0.4922 & 0.9281 & 0.4719 & 0.7649 \\
\hline SSMFN Merged & $\mathbf{0 . 8 1 8 7}$ & 0.7943 & 0.6175 & 0.9442 & 0.6143 & 0.8359 \\
\hline \multicolumn{7}{|c|}{ Validated on The Balanced Validation Dataset } \\
\hline DeepRMethylSite CNN & 0.8090 & 0.8089 & 0.7944 & 0.8251 & 0.6188 & 0.7990 \\
\hline DeepRMethylSite LSTM & 0.7993 & 0.7993 & 0.7618 & 0.8493 & 0.6048 & 0.8024 \\
\hline DeepRMethylSite Merged & 0.8059 & 0.8051 & 0.7659 & 0.8504 & 0.6169 & 0.8021 \\
\hline SMLP & 0.7073 & 0.7073 & 0.7041 & 0.7107 & 0.4147 & 0.7649 \\
\hline SSMFN Merged & $\mathbf{0 . 8 3 6 0}$ & $\mathbf{0 . 8 3 5 8}$ & $\mathbf{0 . 8 1 3 0}$ & 0.8626 & 0.6738 & $\mathbf{0 . 8 3 5 9}$ \\
\hline \multicolumn{7}{|c|}{ Tested on The Test Dataset } \\
\hline $\mathrm{MeMo}^{*}$ & 0.68 & na & 0.38 & 0.99 & 0.46 & na \\
\hline MASA* & 0.65 & na & 0.31 & 0.99 & 0.41 & na \\
\hline BPB-PPMS* & 0.56 & na & 0.12 & 1.00 & 0.25 & na \\
\hline $\mathrm{PMeS}^{*}$ & 0.58 & na & 0.43 & 0.73 & 0.16 & na \\
\hline iMethyl-PseAAC** & 0.59 & na & 0.18 & 1.00 & 0.3 & na \\
\hline PSSMe* & 0.72 & na & 0.6 & 0.83 & 0.44 & na \\
\hline MePred-RF* & 0.69 & na & 0.41 & 0.97 & 0.46 & na \\
\hline PRmePRed** & 0.8683 & na & 0.8709 & 0.8660 & $\mathbf{0 . 7 3 7 0}$ & 0.9000 \\
\hline DeepRMethylSite CNN & 0.7846 & 0.7846 & 0.7803 & 0.7891 & 0.5693 & 0.7846 \\
\hline DeepRMethylSite LSTM & 0.8000 & 0.7989 & 0.7617 & 0.8514 & 0.6065 & 0.8000 \\
\hline DeepRMethylSite Merged & 0.7942 & 0.7929 & 0.7508 & 0.8447 & 0.5959 & 0.7904 \\
\hline SMLP & 0.8077 & 0.8076 & 0.8175 & 0.7985 & 0.6157 & 0.8077 \\
\hline SSMFN Merged & 0.8115 & 0.8115 & 0.8000 & 0.8240 & 0.6235 & 0.8115 \\
\hline
\end{tabular}

Table 6. The First Experiment, Trained on the Balanced Training Dataset

test dataset have different distributions. Because the distribution of methylation is naturally imbalanced, this result suggested that we need to train methylation site prediction models on a dataset with its natural distribution for a practical purpose, not a balanced dataset.

In the second experiment, we trained the models using the imbalanced dataset with a 5 to 1 ratio for negative to positive size samples, respectively. Overall, our model achieved better performance when trained on the imbalanced dataset compared to the balanced dataset. Trained on the imbalanced dataset, SSMFN can even outperform PRmePRed in several metrics. SSMFN accuracy is $0.36 \%$ lower than the DeepRMethylSite accuracy on the imbalanced validation dataset. However, it has better performance on the balanced validation dataset and the test dataset compared to DeepRMethylSite.

\section{CONCLUSIONS}

In general, our proposed model, SSMFN, provided better performance compared to DeepRMethylSite. Our model also performed better when trained on the imbalanced training dataset that it even has better performance than the model that uses feature extraction in several metrics. Additionally, we observed that all the NN models, including ours, achieved a high specificity and a low sensitivity when they were trained on the balanced dataset and tested on the imbalanced dataset. This suggested that, in future works, we need to consider using a dataset with the original distribution for training. This will train the models to recognize the real distribution of the methylation site prediction task, which has far more negative than positive samples, leading to better performance in practice.

\section{ACKNOWLEDGMENTS}

This research is a collaboration between Bioinformatics and Data Science Research Center (BDSRC), Bina Nusantara University and Department of Computer Science, Faculty of Mathematics and Natural 


\begin{tabular}{l|l|l|l|l|l|l}
\hline & Acc & F1 & Sens & Spec & MCC & AUC \\
\hline Model & & \\
\hline \multicolumn{7}{c}{ Validated on The Imbalanced Validation Dataset } \\
\hline DeepRMethylSite CNN & 0.8948 & 0.8550 & 0.9072 & 0.8916 & 0.7242 & 0.8283 \\
\hline DeepRMethylSite LSTM & 0.9092 & 0.8782 & 0.9044 & 0.9106 & 0.7634 & 0.8576 \\
\hline DeepRMethylSite Merged & $\mathbf{0 . 9 1 1 4}$ & $\mathbf{0 . 8 8 0 8}$ & 0.9047 & 0.9115 & $\mathbf{0 . 7 6 9 3}$ & 0.8589 \\
\hline SMLP & 0.9071 & 0.8670 & $\mathbf{0 . 9 9 7 3}$ & 0.8873 & 0.7635 & 0.8295 \\
\hline SSMFN Merged & 0.9078 & 0.8774 & 0.8895 & $\mathbf{0 . 9 1 3 3}$ & 0.7598 & $\mathbf{0 . 8 5 9 6}$ \\
\hline \multicolumn{7}{|c|}{ Validated on The Balanced Validation Dataset } \\
\hline DeepRMethylSite CNN & 0.8289 & 0.8249 & 0.9709 & 0.7527 & 0.6899 & 0.8283 \\
\hline DeepRMethylSite LSTM & 0.8576 & 0.8557 & 0.9644 & 0.7908 & 0.7350 & 0.8576 \\
\hline DeepRMethylSite Merged & 0.8585 & 0.8567 & 0.9645 & 0.7919 & 0.7365 & 0.8589 \\
\hline SMLP & 0.7582 & 0.7432 & $\mathbf{1 . 0 0 0 0}$ & 0.6740 & 0.5899 & 0.8295 \\
\hline SSMFN Merged & $\mathbf{0 . 8 6 5 6}$ & $\mathbf{0 . 8 6 4 0}$ & 0.9672 & $\mathbf{0 . 8 0 0 3}$ & $\mathbf{0 . 7 4 9 1}$ & $\mathbf{0 . 8 5 9 6}$ \\
\hline \multicolumn{7}{|c|}{ Tested on The Test Dataset } \\
\hline DeepRMethylSite CNN & 0.7808 & 0.7727 & 0.9506 & 0.7039 & 0.6063 & 0.7808 \\
\hline DeepRMethylSite LSTM & 0.8115 & 0.8070 & 0.9500 & 0.7382 & 0.6548 & 0.8115 \\
\hline DeepRMethylSite Merged & 0.8135 & 0.8088 & 0.9553 & 0.7390 & 0.6598 & 0.8135 \\
\hline SMLP & 0.7250 & 0.7025 & $\mathbf{1 . 0 0 0 0}$ & 0.6452 & 0.5388 & 0.7250 \\
\hline SSMFN Merged & $\mathbf{0 . 8 4 6 2}$ & $\mathbf{0 . 8 4 3 5}$ & 0.9688 & $\mathbf{0 . 7 7 4 4}$ & $\mathbf{0 . 7 1 7 3}$ & $\mathbf{0 . 8 4 6 2}$ \\
\hline
\end{tabular}

Table 7. Second Experiment, Trained on the Imbalanced Training Dataset

Chen, H., Xue, Y., Huang, N., Yao, X., and Sun, Z. (2006). MeMo: a web tool for prediction of protein methylation modifications. Nucleic Acids Research, 34( $\left.\operatorname{suppl}_{2}\right):$ W249--W253.

${ }_{244}$ Chen, Z., Liu, X., Li, F., Li, C., Marquez-Lago, T., Leier, A., Akutsu, T., Webb, G. I., Xu, D., Smith, A. I., 245 Li, L., Chou, K.-C., and Song, J. (2018). Large-scale comparative assessment of computational predictors 246 for lysine post-translational modification sites. Briefings in Bioinformatics, 20(6):2267-2290.

${ }_{247}$ Chuhan Wu, Fangzhao Wu, Y. C. S. W. Z. Y. Y. H. (2018). Neural metaphor detecting with cnn-lstm model. 248 In Proceedings of the Workshop on Figurative Language Processing, pages 110-114.

${ }_{249}$ Deng, W., Wang, Y., Ma, L., Zhang, Y., Ullah, S., and Xue, Y. (2017). Computational prediction of methyla250 tion types of covalently modified lysine and arginine residues in proteins. Briefings in Bioinformatics, 251 18(4):647-658.

${ }_{252}$ Graves, A. and Schmidhuber, J. (2005). Framewise phoneme classification with bidirectional lstm networks. ${ }_{253}$ In Proceedings. 2005 IEEE International Joint Conference on Neural Networks, 2005., volume 4, pages 254 2047-2052. IEEE.

${ }_{255} \mathrm{Hasan}$, M. M. and Khatun, M. S. (2018). Prediction of protein post-translational modification sites: An 256 overview. Ann Proteom Bioinform, 2:49-57.

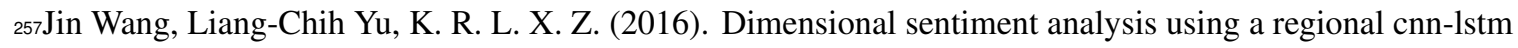


258 259 pages 225-230.

${ }_{260}$ Kumar, P., Joy, J., Pandey, A., and Gupta, D. (2017). Prmepred: A protein arginine methylation prediction 261 tool. PLOS ONE, 12(8):1-12.

${ }_{262}$ Lee, D. Y., Teyssier, C., Strahl, B. D., and Stallcup, M. R. (2005). Role of protein methylation in regulation 263 of transcription. Endocrine Reviews, 26(2):147-170.

${ }_{264} \mathrm{Lee}, \mathrm{T}$.-Y., Chang, C.-W., Lu, C.-T., Cheng, T.-H., and Chang, T.-H. (2014). Identification and charac265 terization of lysine-methylated sites on histones and non-histone proteins. Computational biology and 266 chemistry, 50:11-18.

${ }_{267}$ Lumbanraja, F. R., Mahesworo, B., Cenggoro, T. W., Budiarto, A., and Pardamean, B. (2019). An evaluation 268 of deep neural network performance on limited protein phosphorylation site prediction data. Procedia 269 Computer Science, 157:25-30.

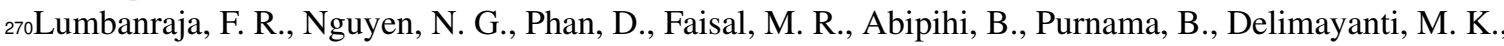
${ }_{271}$ Kubo, M., and Satou, K. (2018). Improved protein phosphorylation site prediction by a new combination 272 of feature set and feature selection. Journal of Biomedical Science and Engineering, 11(6):144-157.

${ }_{273}$ Paszke, A., Gross, S., Massa, F., Lerer, A., Bradbury, J., Chanan, G., Killeen, T., Lin, Z., Gimelshein, N., 274 Antiga, L., Desmaison, A., Köpf, A., Yang, E., DeVito, Z., Raison, M., Tejani, A., Chilamkurthy, S., 275 Steiner, B., Fang, L., Bai, J., and Chintala, S. (2019). Pytorch: An imperative style, high-performance 276 deep learning library. CoRR, abs/1912.01703.

${ }_{277}$ Prabowo, H., Hidayat, A. A., Cenggoro, T. W., Rahutomo, R., Purwandari, K., and Pardamean, B. (2021). 278 Aggregating time series and tabular data in deep learning model for university students' gpa prediction. 279 IEEE Access.

${ }_{280}$ Qiu, W.-R., Xiao, X., Lin, W.-Z., and Chou, K.-C. (2014). iMethyl-PseAAC: identification of protein ${ }_{281}$ methylation sites via a pseudo amino acid composition approach. BioMed research international, 2014. ${ }_{282}$ Schubert, H. L., Blumenthal, R. M., and Cheng, X. (2006). 1 Protein methyltransferases: Their distribution 283 among the five structural classes of AdoMet-dependent methyltransferases. In The Enzymes, volume 24, 284 pages 3-28. Elsevier.

${ }_{285}$ Shao, J., Xu, D., Tsai, S.-N., Wang, Y., and Ngai, S.-M. (2009). Computational identification of protein 286 methylation sites through bi-profile Bayes feature extraction. PloS one, 4(3):e4920.

${ }_{287}$ Shi, S.-P., Qiu, J.-D., Sun, X.-Y., Suo, S.-B., Huang, S.-Y., and Liang, R.-P. (2012). PMeS: prediction of 288 methylation sites based on enhanced feature encoding scheme. PloS one, 7(6):e38772.

${ }_{289}$ Shien, D.-M., Lee, T.-Y., Chang, W.-C., Hsu, J. B.-K., Horng, J.-T., Hsu, P.-C., Wang, T.-Y., and Huang, 290 H.-D. (2009). Incorporating structural characteristics for identification of protein methylation sites. 291 Journal of computational chemistry, 30(9):1532-1543.

${ }_{292}$ Wei, L., Xing, P., Shi, G., Ji, Z.-L., and Zou, Q. (2017). Fast prediction of protein methylation sites using 293 a sequence-based feature selection technique. IEEE/ACM Transactions on Computational Biology and 294 Bioinformatics.

${ }_{295}$ Wen, P.-P., Shi, S.-P., Xu, H.-D., Wang, L.-N., and Qiu, J.-D. (2016). Accurate in silico prediction 296 of species-specific methylation sites based on information gain feature optimization. Bioinformatics, $297 \quad 32(20): 3107-3115$.

${ }_{298} \mathrm{Xu}, \mathrm{Z}$., Li, S., and Deng, W. (2015). Learning temporal features using lstm-cnn architecture for face 299 anti-spoofing. In 2015 3rd IAPR Asian Conference on Pattern Recognition (ACPR), pages 141-145.

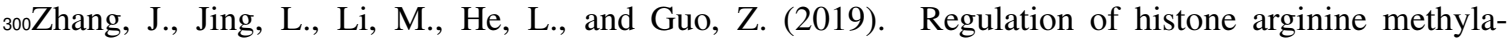
301 tion/demethylation by methylase and demethylase. Molecular Medicine Reports, 19(5):3963-3971. 